\title{
Sustainable Development and Socio-Economic Duality Using Fuzzy System-A Case Study of Iran
}

\author{
Jalil Khodaparast Shirazi ${ }^{1^{*}}$, Mehrzad Ebrahimi ${ }^{1}$, Mazda Moatari ${ }^{2}$ \\ ${ }^{1}$ Department of Economics \& Management, Shiraz Branch, Islamic Azad University, Shiraz, Iran \\ ${ }^{2}$ Sciences and Research Branch, Islamic Azad University, Fars, Iran \\ E-mail: ${ }^{*} J k s @ i a u s h i r a z . n e t$ \\ Received December 20, 2010; revised March 16, 2011; accepted March 28, 2011
}

\begin{abstract}
Homogeneous development and decreasing of inequalities is a logical link between local and national program in macro level. Recognizing inequalities in the process of sustainable development play the major role for programmers. In this study, regarding the complicated qualification and qualitative of socio-economic indices, Iran provinces were ranked by fuzzy system during 2001-2006. In addition, duality in the concept of gap and differences among provinces were determined using the selected indices. Results showed that although country's provinces became more homogeneous from the viewpoint of some of sustainable development indices, the distance among several provinces is still high and there is no clear relation among provinces during this study. This study shows that one development program cannot be effective for all areas of a country with socio-economic duality.
\end{abstract}

Keywords: Sustainable Development, Duality, Economic Index, Health Index, Social Index, Fuzzy System

\section{Introduction}

Reaching development is a relative affair, but attempt to reach this development is definite. How to reach the economic development is a key question for economists and programmers. After World War II, in all countries, developmental strategies and various economic growth models were propounded. To meet the development, resources were destroyed and environmental pollutions were increased. It is in a way that the most important characteristics of development are its potentiality and frequency.

To keep the potentiality of development sources; sustainability should be guaranteed. This is only possible by coordinating socio-economic and environment. Holding United Nation's development conference in 1972 in Sweden which was about environment and keeping its equilibrium was a subject for supporters of environment to try to make economic growth undesirable and confront developed and developing countries. However, it was a rescuer for developing countries but in a symposium, in 1991, the concept of sustainable development investigated, and it was emphasized that sustainable development does not necessarily protect the environment, but it is a new concept of economic growth; a growth which is accompanied by justice and availability of facilities to all people and all the generations without the destruction of limited natural sources of the world.

A special definition of sustainable development was presented in the Johannesburg Summit in 2002. The definition is not in its traditional concept, but known as any process which results in changing human's thoughts preparing them to promote social responsibility [1]. However, how it is possible to increase social responsibility of humans and make a continuous relation among these three sides of triangle: society, economic, and environment. In fact, durable relation between economy of society and environment is a big challenge.

The problem is more serious in developing countries such as Iran regarding un-uniform social and economical characteristics among areas. This study attempts to help effective programming by highlighting the dualities among provinces by using some of social-economic in- dices. In the next step, suggestions for efficient planning have been provided.

\section{Sustainable Development Indices}

Most of social and economic indices directly or indirectly influence each other such as the relationship be- 
tween inflation and felony which has been clearly shown in combinational indices such as HDI. In this research, the merged indices, called socio-economical indices, were used in the study of sustainable development indices.

There are different and variable indices to measure the development. At first, income per capita or GDP per capita were used to show the development, but the re- sults were not satisfactory. In fact, measuring the devel- opment has led to more investigation to find compound indices. On the other hand, compound indices had a compound correlation with income per captia and GDP per capita which resulted in preparing Human Development Index (HDI) (1990) by the United Nations Development Program (UNDP) [2].

Since sustainable development is the commonality of society, different indices have been proposed for each parts of sustainable development: economy and environment in present and future. The important fact is that in sustainable development topic, there is a possibility of recognition between development and sustainable development and also recognition of sustainable development from unsustainable development. UNDP stated 23 social-economic indices for sustainable development [3].

In this study, due to the limitation of preparing statistics for Iranian provinces, 10 indices were selected. These indices were categorized into 4 groups: economic, social, sanitation, and political. To increase the accuracy of calculations, 3 indices close to sustainable indices, including the portion of hospital's bed to 1000 persons, the portion of student to professor, and the inflation rate were added to 7 the previous indices.

\section{Structure of Fuzzy Model}

Basically, although fuzzy systems describe unclear and non-exact phenomenon, fuzzy theory is an exact theory [4]. In fact, the real world is too complicated to present an exact definition of it. Therefore, we should introduce an approximate analytical and acceptable definition. In scientific systems, main information comes from two sources. One of the sources is specialists who define their knowledge about the system with natural language. The second source is the measurements and mathematiccal models, which are derived from the principles of physics. Therefore, the main subject is to combine these two sources of information to design systems. To do so, the key question is how we can formulate knowledge of human beings in a frame similar to mathematical models [4].

In other words, converting human knowledge to mathematical formulae is the main issue. Basically, fuzzy system can address such conversion. Fuzzy rules have been employed to model the deductive phenomena. This will help us to establish structure when unclear and not exact information is available. Then, this structure can be used as a base for predicating, recognizing simul- taneous effects, and further operations.

Therefore, one simple method is to add one fuzzyifier to the input which converts variables with real amount to a fuzzy set, and one defuzzifier which convert a fuzzy set to a variable with real amount in output. After fuzzification on input variables, fuzzy rules will be formed for results. In this step, the logical relation between inputs and outputs are shown which are described in the frame of "if...then" rules. This operation is shown as follows (Figure 1).

\section{Pattern Results}

Ranking the provinces is possible by determining fuzzy system as a pattern and specification of sustainable development indices. It should be mentioned that if 10 selected indices apply once as input, several problems will be emerged. First, the number of fuzzy rules will be increased (for example if each of the inputs would have 3 membership functions, the number of fuzzy rules will be 310). Second, it will decrease the accuracy of calculation. Third, the complexity of operation will be increased and fourth, calculations will be increased.

To eliminate these problems, the 10 selected indices divided to in 4 groups. This classification eliminated the problems and on the other hand, economic, social, and sanitation considered individually. In fact, separate comparison of economic, social, health and political indices provided the chance of new information and stronger analysis.

The first group: economic index. Income per capita, the ratio of exports to GDP, unemployment rate, and inflation rate were categorized as economic indices. Data were obtained from Central Bank of Iran (2001-2006) [5]. In fuzzy rules, it should be considered that an increase in income per capita and export's ratio to GDP of provinces have positive influence on economy, but increasing unemployment rate and inflation rate have negative influences. Figure 2 shows the four above mentioned indices

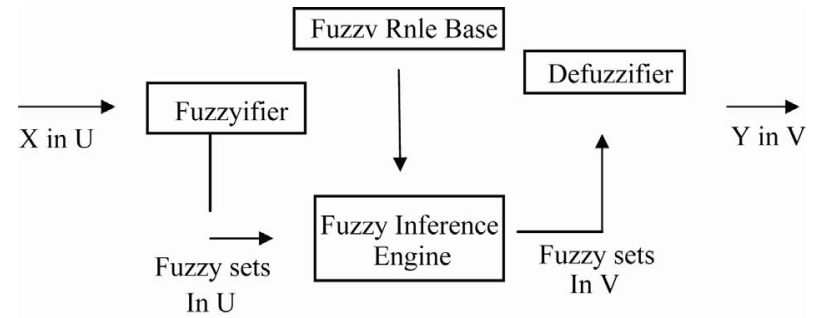

Figure 1. The main structure of fuzzifier and defuzzifier systems. 

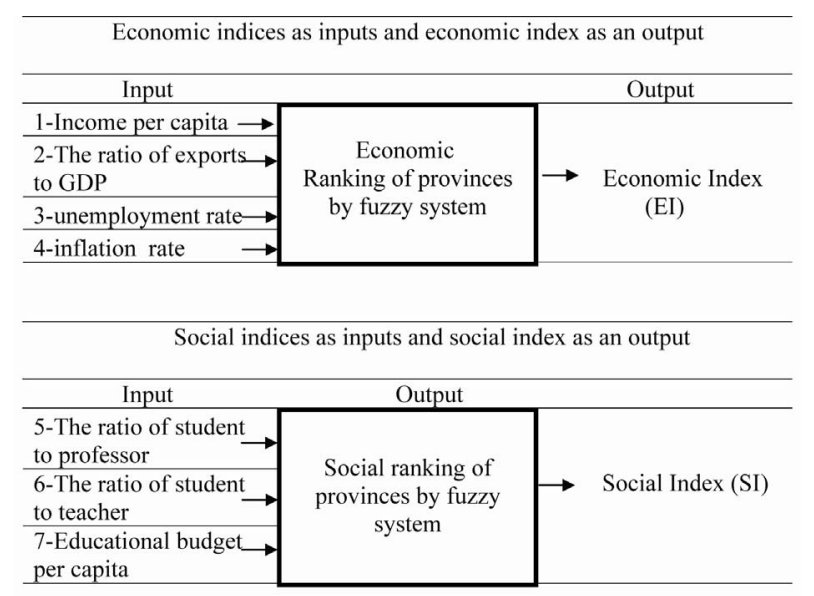

\begin{tabular}{l|l|l}
\hline \multicolumn{2}{c}{ Health indices as inputs and health index as an output } \\
\multicolumn{1}{c}{ Input } \\
$\begin{array}{l}\text { 8-The ratio of } \\
\text { hospital beds per } 1000 \\
\text { persons }\end{array}$ & $\begin{array}{c}\text { Health ranking of } \\
\text { provinces by fuzzy } \\
\text { system }\end{array}$ \\
$\begin{array}{l}\text { 9-health budget per } \rightarrow \\
\text { capita }\end{array}$ & $\rightarrow \begin{array}{c}\text { Health Index } \\
\text { (HI) }\end{array}$ \\
\hline
\end{tabular}

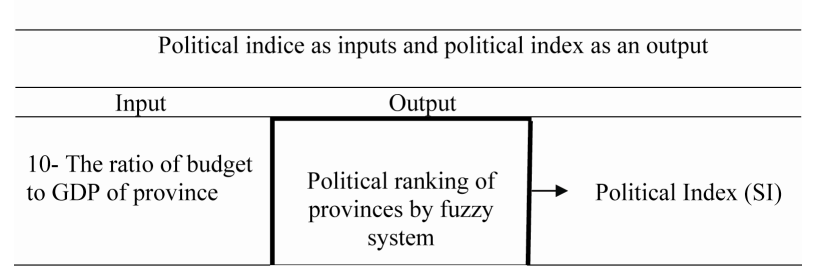

Figure 2. Classification of indices to four groups (economic, social, health, and political indices) and running the fuzzy model separately. Four Indices groups assumed as inputs of fuzzy system and economic, social, health, political indexes as outputs.

as input of fuzzy system and economic index as output for final ranking of provinces (Table 1 and Table 2).

The second group: social index. Indices in this group were included as: the ratio of student to professor, the ratio of student to teacher, and the educational budget per capita. Data were obtained from Central Bank of Iran (2001-2006) [5]. In fuzzy rules, the decrease of student's proportion to professor, student's proportion to teacher, and increase of educational budget per capita have positive influences. Indices were used as input and social index an output for final ranking of provinces. (Figure 2, Table 3 and Table 4).

The Third group: health index. Two indices were placed in this group: ratio of hospital beds per 1000 persons and health budget per capita (Figure 2). Data were obtained from Statistics Canter Iran (2001-2006) [6]. it should be considered that the increase of hospital beds per 1000 persons and health budget per capita have positive influences. Two indices were used as input and
Table 1. Fifty four logical relations of if-then which presents experts opinions by using four selected economic indices. $\mathrm{H}$ (High), M (Medium), L (Low). The output of this Table is used to rank the provinces based on economic index.

\begin{tabular}{|c|c|c|c|c|}
\hline \multicolumn{3}{|c|}{ Input (Economic indices) } & \multicolumn{2}{|r|}{ Output } \\
\hline $\begin{array}{c}\text { Income } \\
\text { per capita }\end{array}$ & $\begin{array}{l}\text { The ratio } \\
\text { of exports } \\
\text { to GDP }\end{array}$ & $\begin{array}{l}\text { Unemployment } \\
\text { rate }\end{array}$ & $\begin{array}{l}\text { Inflation } \\
\text { rate }\end{array}$ & $\begin{array}{l}\text { Economic } \\
\text { Index (EI) }\end{array}$ \\
\hline $\mathrm{L}$ & $\mathrm{L}$ & $\mathrm{L}$ & $\mathrm{L}$ & $\mathrm{L}$ \\
\hline $\mathrm{L}$ & $\mathrm{L}$ & $\mathrm{L}$ & M & $\mathrm{L}$ \\
\hline L & $\mathrm{L}$ & $\mathrm{L}$ & $\mathrm{H}$ & $\mathrm{L}$ \\
\hline $\mathrm{L}$ & $\mathrm{L}$ & $\mathrm{M}$ & $\mathrm{L}$ & $\mathrm{L}$ \\
\hline L & L & $\mathrm{M}$ & $\mathrm{M}$ & $\mathrm{L}$ \\
\hline $\bar{L}$ & $\bar{L}$ & $\mathrm{M}$ & $\mathrm{H}$ & $\mathrm{L}$ \\
\hline $\mathrm{L}$ & $\mathrm{L}$ & $\mathrm{H}$ & $\mathrm{L}$ & $\mathrm{L}$ \\
\hline $\mathrm{L}$ & L & $\mathrm{H}$ & $\mathrm{M}$ & $\mathrm{L}$ \\
\hline $\mathrm{L}$ & $\mathrm{L}$ & $\mathrm{H}$ & $\mathrm{H}$ & $\mathrm{L}$ \\
\hline $\mathrm{L}$ & $\mathrm{H}$ & $\mathrm{L}$ & $\mathrm{L}$ & $\mathrm{M}$ \\
\hline L & $\mathrm{H}$ & $\mathrm{L}$ & $\mathrm{M}$ & $\mathrm{M}$ \\
\hline $\mathrm{L}$ & $\mathrm{H}$ & $\mathrm{L}$ & $\mathrm{H}$ & $\mathrm{M}$ \\
\hline $\mathrm{L}$ & $\mathrm{H}$ & M & L & $\mathrm{M}$ \\
\hline $\mathrm{L}$ & $\mathrm{H}$ & M & $\mathrm{M}$ & M \\
\hline $\mathrm{L}$ & $\mathrm{H}$ & $\mathrm{M}$ & $\mathrm{H}$ & $\mathrm{L}$ \\
\hline $\mathrm{L}$ & $\mathrm{H}$ & $\mathrm{H}$ & L & $\mathrm{L}$ \\
\hline $\mathrm{L}$ & $\mathrm{H}$ & $\mathrm{H}$ & $\mathrm{M}$ & $\mathrm{L}$ \\
\hline $\mathrm{L}$ & $\mathrm{H}$ & $\mathrm{H}$ & $\mathrm{H}$ & $\mathrm{L}$ \\
\hline M & $\mathrm{L}$ & $\mathrm{L}$ & $\mathrm{L}$ & M \\
\hline $\mathrm{M}$ & $\mathrm{L}$ & $\mathrm{L}$ & $\mathrm{M}$ & $\mathrm{M}$ \\
\hline $\mathrm{M}$ & L & $\mathrm{L}$ & $\mathrm{H}$ & $\mathrm{M}$ \\
\hline M & $\mathrm{L}$ & M & $\mathrm{L}$ & M \\
\hline M & $\mathrm{L}$ & M & M & M \\
\hline M & $\mathrm{L}$ & M & $\mathrm{H}$ & M \\
\hline M & $\mathrm{L}$ & $\mathrm{H}$ & $\mathrm{L}$ & $\mathrm{L}$ \\
\hline M & $\mathrm{L}$ & $\mathrm{H}$ & M & $\mathrm{L}$ \\
\hline M & $\mathrm{L}$ & $\mathrm{H}$ & $\mathrm{H}$ & $\mathrm{L}$ \\
\hline M & $\mathrm{H}$ & $\mathrm{L}$ & $\mathrm{L}$ & $\mathrm{H}$ \\
\hline M & $\mathrm{H}$ & $\mathrm{L}$ & M & M \\
\hline M & $\mathrm{H}$ & $\mathrm{L}$ & $\mathrm{H}$ & M \\
\hline M & $\mathrm{H}$ & M & $\mathrm{L}$ & $\mathrm{H}$ \\
\hline M & $\mathrm{H}$ & M & M & M \\
\hline M & $\mathrm{H}$ & M & $\mathrm{H}$ & $\mathrm{L}$ \\
\hline $\mathrm{M}$ & $\mathrm{H}$ & $\mathrm{H}$ & $\mathrm{L}$ & $\mathrm{M}$ \\
\hline $\mathrm{M}$ & $\mathrm{H}$ & $\mathrm{H}$ & $\mathrm{M}$ & $\mathrm{L}$ \\
\hline $\mathrm{M}$ & $\mathrm{H}$ & $\mathrm{H}$ & $\mathrm{H}$ & $\mathrm{L}$ \\
\hline $\mathrm{H}$ & $\mathrm{L}$ & $\mathrm{L}$ & $\mathrm{L}$ & $\mathrm{H}$ \\
\hline $\mathrm{H}$ & $\mathrm{L}$ & $\mathrm{L}$ & $\mathrm{M}$ & $\mathrm{H}$ \\
\hline $\mathrm{H}$ & $\mathrm{L}$ & $\mathrm{L}$ & $\mathrm{H}$ & $\mathrm{H}$ \\
\hline $\mathrm{H}$ & $\mathrm{L}$ & $\mathrm{M}$ & $\mathrm{L}$ & $\mathrm{M}$ \\
\hline $\mathrm{H}$ & $\mathrm{L}$ & $\mathrm{M}$ & $\mathrm{M}$ & $\mathrm{M}$ \\
\hline $\mathrm{H}$ & $\mathrm{L}$ & $\mathrm{M}$ & $\mathrm{H}$ & $\mathrm{M}$ \\
\hline $\mathrm{H}$ & $\mathrm{L}$ & $\mathrm{H}$ & $\mathrm{L}$ & $\mathrm{L}$ \\
\hline $\mathrm{H}$ & $\mathrm{L}$ & $\mathrm{H}$ & $\mathrm{M}$ & $\mathrm{L}$ \\
\hline $\mathrm{H}$ & $\mathrm{L}$ & $\mathrm{H}$ & $\mathrm{H}$ & $\mathrm{L}$ \\
\hline $\mathrm{H}$ & $\mathrm{H}$ & $\mathrm{L}$ & $\mathrm{L}$ & $\mathrm{H}$ \\
\hline $\mathrm{H}$ & $\mathrm{H}$ & $\mathrm{L}$ & $\mathrm{M}$ & $\mathrm{H}$ \\
\hline $\mathrm{H}$ & $\mathrm{H}$ & $\mathrm{L}$ & $\mathrm{H}$ & $\mathrm{H}$ \\
\hline $\mathrm{H}$ & $\mathrm{H}$ & $\mathrm{M}$ & $\mathrm{L}$ & $\mathrm{H}$ \\
\hline $\mathrm{H}$ & $\mathrm{H}$ & $\mathrm{M}$ & $\mathrm{M}$ & $\mathrm{H}$ \\
\hline $\mathrm{H}$ & $\mathrm{H}$ & $\mathrm{M}$ & $\mathrm{H}$ & $\mathrm{M}$ \\
\hline $\mathrm{H}$ & $\mathrm{H}$ & $\mathrm{H}$ & $\mathrm{L}$ & $\mathbf{M}$ \\
\hline $\mathrm{H}$ & $\mathrm{H}$ & $\mathrm{H}$ & $\mathrm{M}$ & $\mathrm{L}$ \\
\hline $\mathrm{H}$ & $\mathrm{H}$ & $\mathrm{H}$ & $\mathrm{H}$ & $\mathrm{L}$ \\
\hline
\end{tabular}


Table 2. Ranking of provinces from the viewpoint of economic index. Output of this Table is one of the inputs for final ranking of provinces.

\begin{tabular}{|c|c|c|c|c|c|c|}
\hline \multirow{2}{*}{ Province } & \multicolumn{6}{|c|}{ Year } \\
\hline & 2001 & 2002 & 2003 & 2004 & 2005 & 2006 \\
\hline Azarbayjan Sharghi & 0.1 & 0.11 & 0.15 & 0.31 & 0.4 & 0.45 \\
\hline Ardebil & 0.1 & 0.1 & 0.1 & 0.14 & 0.19 & 0.37 \\
\hline Isfahan & 0.47 & 0.46 & 0.43 & 0.46 & 0.46 & 0.48 \\
\hline Ilam & 0.1 & 0.1 & 0.12 & 0.44 & 0.49 & 0.1 \\
\hline Tehran & 0.29 & 0.42 & 0.46 & 0.47 & 0.49 & 0.5 \\
\hline Chaharmahal Bakhtiari & 0.1 & 0.1 & 0.1 & 0.11 & 0.17 & 0.35 \\
\hline Khorasan & 0.1 & 0.12 & 0.17 & 0.18 & 0.33 & 0.43 \\
\hline Khuzestan & 0.46 & 0.18 & 0.42 & 0.47 & 0.52 & 0.45 \\
\hline Zanjan & 0.46 & 0.42 & 0.32 & 0.18 & 0.37 & 0.45 \\
\hline Semnan & 0.11 & 0.16 & 0.29 & 0.45 & 0.46 & 0.48 \\
\hline Sistan Baluchestan & 0.1 & 0.1 & 0.1 & 0.1 & 0.1 & 0.1 \\
\hline Fars & 0.1 & 0.11 & 0.15 & 0.27 & 0.39 & 0.45 \\
\hline Qom & 0.1 & 0.1 & 0.13 & 0.23 & 0.36 & 0.43 \\
\hline Kordestan & 0.1 & 0.1 & 0.12 & 0.31 & 0.42 & 0.33 \\
\hline Kerman & 0.46 & 0.45 & 0.48 & 0.2 & 0.41 & 0.2 \\
\hline Kermanshah & 0.1 & 0.1 & 0.36 & 0.18 & 0.44 & 0.12 \\
\hline Kohgiluye Boyrahmad & 0.5 & 0.5 & 0.51 & 0.5 & 0.46 & 0.4 \\
\hline Golestan & 0.1 & 0.1 & 0.11 & 0.17 & 0.24 & 0.37 \\
\hline Gilan & 0.16 & 0.1 & 0.14 & 0.19 & 0.35 & 0.43 \\
\hline Lorestan & 0.1 & 0.1 & 0.1 & 0.1 & 0.15 & 0.1 \\
\hline Mazandaran & 0.1 & 0.14 & 0.23 & 0.38 & 0.44 & 0.46 \\
\hline Markazi & 0.35 & 0.35 & 0.41 & 0.46 & 0.47 & 0.49 \\
\hline Hormozgan & 0.47 & 0.14 & 0.2 & 0.44 & 0.5 & 0.5 \\
\hline Hamedan & 0.1 & 0.1 & 0.1 & 0.16 & 0.23 & 0.4 \\
\hline Yazd & 0.1 & 0.15 & 0.22 & 0.36 & 0.45 & 0.47 \\
\hline
\end{tabular}

Table 3. Twenty seven logical relation of if-then which presents experts opinions by using 3 selected social indices. $H$ (High), M (Medium), L (Low). The output of this table (Social Index, SI) is used for final ranking of provinces socially.

\begin{tabular}{|c|c|c|c|}
\hline \multicolumn{2}{|c|}{ Input (Social indices) } & \multirow[b]{2}{*}{ Educational budget per capita } & \multirow{2}{*}{$\begin{array}{c}\text { Output } \\
\text { Social Index (SI) }\end{array}$} \\
\hline The ratio of student to professor & The ratio of student to teacher & & \\
\hline $\mathrm{L}$ & $\mathrm{L}$ & $\mathrm{L}$ & M \\
\hline $\mathrm{L}$ & $\mathrm{L}$ & M & $\mathrm{H}$ \\
\hline $\mathrm{L}$ & $\mathrm{L}$ & $\mathrm{H}$ & $\mathrm{H}$ \\
\hline $\mathrm{L}$ & M & $\mathrm{L}$ & M \\
\hline $\mathrm{L}$ & M & M & M \\
\hline $\mathrm{L}$ & $\mathrm{H}$ & $\mathrm{L}$ & $\mathrm{L}$ \\
\hline $\mathrm{L}$ & $\mathrm{H}$ & M & $\mathrm{L}$ \\
\hline $\mathrm{L}$ & $\mathrm{H}$ & $\mathrm{H}$ & M \\
\hline M & $\mathrm{L}$ & $\mathrm{L}$ & $\mathrm{L}$ \\
\hline M & $\mathrm{L}$ & M & M \\
\hline M & $\mathrm{L}$ & $\mathrm{H}$ & $\mathrm{H}$ \\
\hline M & M & $\mathrm{L}$ & M \\
\hline M & M & M & M \\
\hline M & $\mathrm{H}$ & $\mathrm{L}$ & $\mathrm{L}$ \\
\hline M & $\mathrm{H}$ & M & $\mathrm{L}$ \\
\hline $\mathrm{M}$ & $\mathrm{H}$ & $\mathrm{H}$ & $\mathrm{M}$ \\
\hline $\mathrm{H}$ & $\mathrm{L}$ & $\mathrm{L}$ & $\mathrm{L}$ \\
\hline $\mathrm{H}$ & $\mathrm{L}$ & M & $\mathrm{M}$ \\
\hline $\mathrm{H}$ & $\mathrm{L}$ & $\mathrm{H}$ & $\mathrm{M}$ \\
\hline $\mathrm{H}$ & $\mathrm{M}$ & $\mathrm{L}$ & $\mathrm{L}$ \\
\hline $\mathrm{H}$ & $\mathrm{M}$ & $\mathrm{M}$ & $\mathrm{M}$ \\
\hline $\mathrm{H}$ & $\mathrm{M}$ & $\mathrm{H}$ & $\mathrm{M}$ \\
\hline $\mathrm{H}$ & $\mathrm{H}$ & $\mathrm{L}$ & $\mathrm{L}$ \\
\hline $\mathrm{H}$ & $\mathrm{H}$ & $\mathrm{M}$ & $\mathrm{L}$ \\
\hline $\mathrm{H}$ & $\mathrm{H}$ & $\mathrm{H}$ & $\mathrm{L}$ \\
\hline
\end{tabular}


Table 4. Ranking of provinces from the viewpoint of social index. Output of this table (social index) is one of the inputs for final ranking of provinces.

\begin{tabular}{|c|c|c|c|c|c|c|}
\hline \multirow{2}{*}{ Province } & \multicolumn{6}{|c|}{ Year } \\
\hline & 2001 & 2002 & 2003 & 2004 & 2005 & 2006 \\
\hline Azarbayjan Shargh & 0.5 & 0.5 & 0.48 & 0.47 & 0.52 & 0.56 \\
\hline Azarbayja Gharbi & 0.45 & 0.5 & 0.5 & 0.27 & 0.5 & 0.53 \\
\hline Ardebil & 0.33 & 0.44 & 0.48 & 0.47 & 0.5 & 0.52 \\
\hline Isfahan & 0.5 & 0.49 & 0.47 & 0.43 & 0.54 & 0.6 \\
\hline Ilam & 0.42 & 0.48 & 0.44 & 0.24 & 0.84 & 0.87 \\
\hline Bushehr & 0.15 & 0.19 & 0.39 & 0.27 & 0.52 & 0.54 \\
\hline Tehran & 0.19 & 0.4 & 0.49 & 0.5 & 0.5 & 0.51 \\
\hline Chaharmahal Bakhtiari & 0.49 & 0.17 & 0.48 & 0.42 & 0.65 & 0.87 \\
\hline Khorasan & 0.5 & 0.49 & 0.46 & 0.36 & 0.43 & 0.64 \\
\hline Khuzestan & 0.12 & 0.12 & 0.14 & 0.19 & 0.5 & 0.58 \\
\hline Zanjan & 0.33 & 0.27 & 0.4 & 0.48 & 0.51 & 0.75 \\
\hline Semnan & 0.48 & 0.47 & 0.4 & 0.46 & 0.57 & 0.7 \\
\hline Sistan Baluchestan & 0.12 & 0.12 & 0.12 & 0.12 & 0.16 & 0.33 \\
\hline Fars & 0.48 & 0.48 & 0.5 & 0.49 & 0.53 & 0.58 \\
\hline Qazvin & 0.44 & 0.45 & 0.5 & 0.5 & 0.51 & 0.54 \\
\hline Qom & 0.47 & 0.5 & 0.5 & 0.46 & 0.54 & 0.56 \\
\hline Kordestan & 0.3 & 0.22 & 0.47 & 0.42 & 0.52 & 0.64 \\
\hline Kerman & 0.5 & 0.49 & 0.47 & 0.45 & 0.53 & 0.69 \\
\hline Kermanshah & 0.44 & 0.5 & 0.5 & 0.48 & 0.54 & 0.85 \\
\hline Kohgiluye Boyrahmad & 0.2 & 0.12 & 0.12 & 0.45 & 0.6 & 0.64 \\
\hline Golestan & 0.49 & 0.46 & 0.44 & 0.47 & 0.51 & 0.55 \\
\hline Gilan & 0.29 & 0.22 & 0.19 & 0.33 & 0.57 & 0.81 \\
\hline Lorestan & 0.27 & 0.44 & 0.5 & 0.45 & 0.5 & 0.51 \\
\hline Mazandaran & 0.24 & 0.19 & 0.15 & 0.17 & 0.52 & 0.86 \\
\hline Markazi & 0.49 & 0.5 & 0.49 & 0.5 & 0.52 & 0.56 \\
\hline Hormozgan & 0.12 & 0.12 & 0.12 & 0.12 & 0.42 & 0.5 \\
\hline Hamedan & 0.5 & 0.5 & 0.5 & 0.49 & 0.53 & 0.7 \\
\hline Yazd & 0.26 & 0.2 & 0.28 & 0.19 & 0.54 & 0.56 \\
\hline
\end{tabular}

health index as output. (Figure 2, Table 5 and Table 6).

The Fourth group: political index. In this group, only the ratio of budget to GDP of provinces which has been considered as an interference index of government was considered (Figure 2). Data was obtained from The Management and Planning Organization of Iran (MPO) [7]. As a result, there was just two fuzzy sets (high and low) for the political index (Table 7). In making fuzzy rules, the increase of government's interference has negative influence on social and economic indices. Since there was only one index here, there was no need to make separate fuzzy system for political index (Table 7).

\section{Fuzzy System Phases}

After ranking 10 selected indices to economic, social, health and political, at first 3 fuzzy systems were carried out for 3 groups (economic, social, and health) by Matlab software. Then, for each inputs, three fuzzy sets, including high, medium, and low were allocated (Table 1, Table 3, Table 5 and Table 7). There was 2 exceptions, including: the ratio of export to GDP and the index of government's interference which 2 fuzzy set of high and low were considered (Table 1 and Table 7). For economic group, 54 rule bases were generated, while, 27, 9,

Table 5. Nine logical relations of if-then which presents 2 selected health indices by using experts opinions and information. H (High), M (Medium), L (Low). The output of this Table (Health Index, HI) is used to rank the provinces from the view point of health index.

\begin{tabular}{ccc}
\hline \multicolumn{2}{c}{ Input (Health indices) } & Output \\
\hline $\begin{array}{c}\text { The ratio of hospital beds } \\
\text { per } 1000 \text { person }\end{array}$ & $\begin{array}{c}\text { Health budget per } \\
\text { capita }\end{array}$ & $\begin{array}{c}\text { Health Index } \\
\text { (HI) }\end{array}$ \\
\hline L & L & L \\
L & M & H \\
L & H & H \\
M & L & L \\
M & M & H \\
M & H & H \\
H & L & L \\
H & M & H \\
H & H & H \\
\hline
\end{tabular}


Table 6. Ranking of provinces from the viewpoint of health index. Output of this Table is one of the inputs for final ranking of provinces.

\begin{tabular}{|c|c|c|c|c|c|c|}
\hline \multirow{2}{*}{ Province } & \multicolumn{6}{|c|}{ Year } \\
\hline & 2001 & 2002 & 2003 & 2004 & 2005 & 2006 \\
\hline Azarbayjan Sharghi & 0.26 & 0.26 & 0.26 & 0.26 & 0.26 & 0.26 \\
\hline Azarbayjan Gharbi & 0.26 & 0.26 & 0.26 & 0.26 & 0.27 & 0.27 \\
\hline Ardebil & 0.74 & 0.74 & 0.74 & 0.74 & 0.26 & 0.26 \\
\hline Isfahan & 0.34 & 0.36 & 0.37 & 0.39 & 0.26 & 0.29 \\
\hline Ilam & 0.74 & 0.74 & 0.74 & 0.74 & 0.74 & 0.74 \\
\hline Bushehr & 0.74 & 0.74 & 0.74 & 0.74 & 0.74 & 0.74 \\
\hline Tehran & 0.33 & 0.33 & 0.34 & 0.34 & 0.26 & 0.26 \\
\hline Chaharmahal Bakhtiari & 0.74 & 0.74 & 0.74 & 0.74 & 0.72 & 0.74 \\
\hline Khorasan & 0.74 & 0.74 & 0.74 & 0.74 & 0.28 & 0.72 \\
\hline Khuzestan & 0.26 & 0.26 & 0.26 & 0.26 & 0.26 & 0.28 \\
\hline Zanjan & 0.74 & 0.74 & 0.74 & 0.74 & 0.26 & 0.26 \\
\hline Semnan & 0.74 & 0.74 & 0.74 & 0.74 & 0.26 & 0.38 \\
\hline Sistan Baluchestan & 0.74 & 0.74 & 0.74 & 0.74 & 0.26 & 0.73 \\
\hline Fars & 0.68 & 0.7 & 0.71 & 0.72 & 0.26 & 0.59 \\
\hline Qazvin & 0.7 & 0.71 & 0.71 & 0.72 & 0.26 & 0.34 \\
\hline Qom & 0.36 & 0.37 & 0.39 & 0.4 & 0.57 & 0.74 \\
\hline Kordestan & 0.74 & 0.74 & 0.74 & 0.74 & 0.74 & 0.39 \\
\hline Kerman & 0.72 & 0.72 & 0.72 & 0.73 & 0.74 & 0.74 \\
\hline Kermanshah & 0.26 & 0.26 & 0.26 & 0.26 & 0.74 & 0.74 \\
\hline Kohgiluye Boyrahmad & 0.74 & 0.74 & 0.74 & 0.74 & 0.74 & 0.74 \\
\hline Golestan & 0.74 & 0.74 & 0.74 & 0.74 & 0.26 & 0.73 \\
\hline Gilan & 0.66 & 0.68 & 0.7 & 0.71 & 0.26 & 0.66 \\
\hline Lorestan & 0.74 & 0.74 & 0.74 & 0.74 & 0.26 & 0.66 \\
\hline Mazandaran & 0.26 & 0.26 & 0.26 & 0.26 & 0.27 & 0.67 \\
\hline Markazi & 0.66 & 0.68 & 0.69 & 0.71 & 0.72 & 0.64 \\
\hline Hormozgan & 0.74 & 0.74 & 0.74 & 0.74 & 0.71 & 0.69 \\
\hline Hamedan & 0.74 & 0.74 & 0.74 & 0.74 & 0.27 & 0.63 \\
\hline Yazd & 0.74 & 0.74 & 0.74 & 0.74 & 0.65 & 0.74 \\
\hline
\end{tabular}

Table 7. Two logical relations of if-then of the ratio of budget to province's GDP. H (High), L (Low). Its two outputs were used as a political index and as an input for final ranking of provinces.

\begin{tabular}{ll}
\hline Input & Output \\
\hline The ratio of budget to province's GDP & Political Index (PI) \\
L & L \\
H & H \\
\hline
\end{tabular}

and 2 rule bases were obtained for social health, and political groups respectively (Table $\mathbf{1}$, Table 3 , Table 5 , and Table 7). Their outputs made economic, social, health, and political indices (Table 1, Table 3, Table 5, and Table 7).

The obtained results were employed as inputs of the second phase fuzzy system for the last ranking of provinces which totally produced 36 rule bases (Table 8). In the next phase, the generated output was a number be tween zero and one, providing a practical quantity to compare provinces. The fuzzy system made 3 outputs for economic group, 2 outputs for social group, 2 for health, and 2 for political group which are summarized in Table 9. The result of these outputs were 36 cases indicating 36 relation of "if...then" between inputs and outputs of system. These rules were obtained by opinions and experiences of experts (Table 9). By using MAT$\mathrm{LAB}$ software and entering information from 2001 to 
Table 8. shows 36 logical relation of if- then which uses expert's opinions and information and uses socio, economic, health and political indices. $\mathrm{P}$ (positive effect), $\mathbf{N}$ (negative effect). The output of this table is used for final ranking of provinces.

\begin{tabular}{|c|c|c|c|c|c|}
\hline & \multicolumn{4}{|c|}{ Inputs } & \multirow[t]{2}{*}{ Outputs } \\
\hline & $\mathbf{P}$ & $\mathbf{P}$ & $\mathbf{P}$ & $\mathbf{N}$ & \\
\hline Rule-Base & EI & SI & HI & PI & $\mathrm{TI}$ \\
\hline 1 & $\mathrm{~L}$ & $\mathrm{~L}$ & $\mathrm{~L}$ & $\mathrm{H}$ & V V V L \\
\hline 2 & $\mathrm{~L}$ & $\mathrm{~L}$ & $\mathrm{~L}$ & $\mathrm{~L}$ & V V V L \\
\hline 3 & $\mathrm{~L}$ & $\mathrm{~L}$ & $\mathrm{H}$ & $\mathrm{H}$ & V V L \\
\hline 4 & $\mathrm{~L}$ & $\mathrm{~L}$ & $\mathrm{H}$ & $\mathrm{L}$ & V V L \\
\hline 5 & $\mathrm{~L}$ & M & $\mathrm{L}$ & $\mathrm{H}$ & V V L \\
\hline 6 & $\mathrm{~L}$ & M & $\mathrm{L}$ & $\mathrm{L}$ & V L \\
\hline 7 & $\mathrm{~L}$ & $\mathrm{M}$ & $\mathrm{H}$ & $\mathrm{H}$ & V L \\
\hline 8 & $\mathrm{~L}$ & $\mathrm{M}$ & $\mathrm{H}$ & $\mathrm{L}$ & V L \\
\hline 9 & $\mathrm{~L}$ & $\mathrm{H}$ & $\mathrm{L}$ & $\mathrm{H}$ & V L \\
\hline 10 & $\mathrm{~L}$ & $\mathrm{H}$ & $\mathrm{L}$ & $\mathrm{L}$ & $\mathrm{L}$ \\
\hline 11 & $\mathrm{~L}$ & $\mathrm{H}$ & $\mathrm{H}$ & $\mathrm{H}$ & $\mathrm{L}$ \\
\hline 12 & $\mathrm{~L}$ & $\mathrm{H}$ & $\mathrm{H}$ & $\mathrm{L}$ & L \\
\hline 13 & M & $\mathrm{L}$ & $\mathrm{L}$ & $\mathrm{H}$ & $\mathrm{L}$ \\
\hline 14 & M & $\mathrm{L}$ & $\mathrm{L}$ & $\mathrm{L}$ & $\mathrm{L}$ \\
\hline 15 & M & $\mathrm{L}$ & $\mathrm{H}$ & $\mathrm{H}$ & M \\
\hline 16 & M & $\mathrm{L}$ & $\mathrm{H}$ & $\mathrm{L}$ & M \\
\hline 17 & M & M & $\mathrm{L}$ & $\mathrm{H}$ & M \\
\hline 18 & M & M & $\mathrm{L}$ & $\mathrm{L}$ & M \\
\hline 19 & M & M & $\mathrm{H}$ & $\mathrm{H}$ & M \\
\hline 20 & M & M & $\mathrm{H}$ & $\mathrm{L}$ & M \\
\hline 21 & M & $\mathrm{H}$ & $\mathrm{L}$ & $\mathrm{H}$ & M \\
\hline 22 & M & $\mathrm{H}$ & $\mathrm{L}$ & $\mathrm{L}$ & M \\
\hline 23 & M & $\mathrm{H}$ & $\mathrm{H}$ & $\mathrm{H}$ & $\mathrm{H}$ \\
\hline 24 & M & $\mathrm{H}$ & $\mathrm{H}$ & $\mathrm{L}$ & $\mathrm{H}$ \\
\hline 25 & $\mathrm{H}$ & $\mathrm{L}$ & $\mathrm{L}$ & $\mathrm{H}$ & $\mathrm{H}$ \\
\hline 26 & $\mathrm{H}$ & $\mathrm{L}$ & $\mathrm{L}$ & $\mathrm{L}$ & $\mathrm{H}$ \\
\hline 27 & $\mathrm{H}$ & $\mathrm{L}$ & $\mathrm{H}$ & $\mathrm{H}$ & $\mathrm{H}$ \\
\hline 28 & $\mathrm{H}$ & $\mathrm{L}$ & $\mathrm{H}$ & $\mathrm{L}$ & V H \\
\hline 29 & $\mathrm{H}$ & M & $\mathrm{L}$ & $\mathrm{H}$ & V H \\
\hline 30 & $\mathrm{H}$ & M & $\mathrm{L}$ & $\mathrm{L}$ & V H \\
\hline 31 & $\mathrm{H}$ & M & $\mathrm{H}$ & $\mathrm{H}$ & V H \\
\hline 32 & $\mathrm{H}$ & M & $\mathrm{H}$ & $\mathrm{L}$ & V V H \\
\hline 33 & $\mathrm{H}$ & $\mathrm{H}$ & $\mathrm{L}$ & $\mathrm{H}$ & V V H \\
\hline 34 & $\mathrm{H}$ & $\mathrm{H}$ & $\mathrm{L}$ & $\mathrm{L}$ & V V H \\
\hline 35 & $\mathrm{H}$ & $\mathrm{H}$ & $\mathrm{H}$ & $\mathrm{H}$ & V V V H \\
\hline 36 & $\mathrm{H}$ & $\mathrm{H}$ & $\mathrm{H}$ & $\mathrm{L}$ & V V V H \\
\hline
\end{tabular}


Table 9. Classification of indices and entrances of second phase of fuzzy system, H (High), M (Medium), L (Low).

\begin{tabular}{|c|c|c|c|c|c|}
\hline Group & Entrance & $\begin{array}{l}\text { Fuzzy Sets } \\
\text { First stage }\end{array}$ & $\begin{array}{l}\text { Rule } \\
\text { Base }\end{array}$ & $\begin{array}{l}\text { System's output or } \\
\text { entrance to rank the } \\
\text { provinces }\end{array}$ & $\begin{array}{l}\text { Fuzzy sets } \\
\text { second stage }\end{array}$ \\
\hline Economic & $\begin{array}{l}\text { 1-Income pre capita } \\
\text { 2-The ratio of exports to GDP } \\
\text { 3-Unemployment rate } \\
\text { 4-Inflation rate }\end{array}$ & $\begin{array}{l}\text { H M L } \\
\text { H L } \\
\text { H M L } \\
\text { HM L }\end{array}$ & $\begin{array}{c}54 \\
\text { cases }\end{array}$ & Economic Index (EI) & H M L \\
\hline Social & $\begin{array}{l}\text { 5-The ratio of student to professor } \\
\text { 6-The ratio of student to teacher } \\
\text { 7-Educational budget per capita }\end{array}$ & $\begin{array}{l}\text { H M L } \\
\text { H M L } \\
\text { H M L }\end{array}$ & $\begin{array}{c}27 \\
\text { cases }\end{array}$ & $\begin{array}{l}\text { Social Index } \\
\text { (SI) }\end{array}$ & H M L \\
\hline Health & $\begin{array}{l}\text { 8-The ratio of hospital beds per } 1000 \text { persons } \\
\text { 9-Health budget per capita }\end{array}$ & $\begin{array}{l}\text { H M L } \\
\text { H M L }\end{array}$ & $\begin{array}{c}9 \\
\text { cases }\end{array}$ & $\begin{array}{l}\text { Health Index } \\
\text { (HI) }\end{array}$ & H L \\
\hline Political & $\begin{array}{l}\text { 10-Government's interference (budget divided to } \\
\text { GDP) }\end{array}$ & H L & $\begin{array}{c}2 \\
\text { cases }\end{array}$ & $\begin{array}{l}\text { Political Index } \\
\text { (PI) }\end{array}$ & $\mathrm{H} \mathrm{L}$ \\
\hline
\end{tabular}

2006 of economical, social, health, and political indices, from 2001 to 2006 the final ranking was obtained (Table 10).

\section{Considerable Differences and Inequalities between Provinces}

In this study, provinces of Iran were compared and ranked in the view of some sustainable development in- dices with fuzzy system as a model of developing country with un-uniform areas. The obtained results revealed high distance and difference among provinces of country according to the above indices (Table 10). Similar to overall indices, the differences were also observed in each of social, economic and health groups (Table 2, Table 4, and Table 6). This finding is in line with previous observations of Noorbakhsh [8] and Ebrahimi [9].

It should be mentioned that during the investigation

Table 10. Ranking of different provinces of Iran (2001-2006) by fuzzy system.

\begin{tabular}{|c|c|c|c|c|c|c|}
\hline \multirow{2}{*}{$\begin{array}{ll}\text { Province } & \text { Year } \\
\end{array}$} & \multicolumn{6}{|c|}{ Total Ranking } \\
\hline & 2001 & 2002 & 2003 & 2004 & 2005 & 2006 \\
\hline Azarbayjan Sharghi & 0.23 & 0.23 & 0.24 & 0.44 & 0.5 & 0.5 \\
\hline Azarbayjan Gharbi & 0.19 & 0.16 & 0.18 & 0.17 & 0.2 & 0.41 \\
\hline Ardebil & 0.23 & 0.24 & 0.24 & 0.25 & 0.17 & 0.49 \\
\hline Isfahan & 0.5 & 0.5 & 0.5 & 0.5 & 0.5 & 0.5 \\
\hline Ilam & 0.24 & 0.24 & 0.24 & 0.49 & 0.62 & 0.37 \\
\hline Tehran & 0.34 & 0.5 & 0.5 & 0.5 & 0.5 & 0.5 \\
\hline Chaharmahal Bakhtiari & 0.24 & 0.13 & 0.24 & 0.24 & 0.26 & 0.6 \\
\hline Khorasan & 0.24 & 0.24 & 0.26 & 0.26 & 0.45 & 0.51 \\
\hline Khuzestan & 0.38 & 0.09 & 0.38 & 0.39 & 0.5 & 0.5 \\
\hline Zanjan & 0.5 & 0.49 & 0.46 & 0.26 & 0.48 & 0.51 \\
\hline Samnan & 0.24 & 0.25 & 0.42 & 0.5 & 0.5 & 0.52 \\
\hline Fars & 0.23 & 0.24 & 0.25 & 0.33 & 0.49 & 0.5 \\
\hline Qazvin & 0.25 & 0.26 & 0.29 & 0.5 & 0.5 & 0.5 \\
\hline Qom & 0.22 & 0.22 & 0.24 & 0.27 & 0.48 & 0.5 \\
\hline Kordestan & 0.21 & 0.15 & 0.24 & 0.45 & 0.5 & 0.46 \\
\hline Kerman & 0.5 & 0.5 & 0.5 & 0.26 & 0.5 & 0.31 \\
\hline Kermanshah & 0.13 & 0.13 & 0.48 & 0.18 & 0.5 & 0.37 \\
\hline Kohgiluye Boyrahmad & 0.49 & 0.49 & 0.49 & 0.5 & 0.5 & 0.51 \\
\hline Golestan & 0.24 & 0.24 & 0.24 & 0.26 & 0.24 & 0.49 \\
\hline Gilan & 0.22 & 0.14 & 0.14 & 0.25 & 0.47 & 0.61 \\
\hline Lorestan & 0.18 & 0.24 & 0.24 & 0.24 & 0.15 & 0.23 \\
\hline Mazandaran & 0.16 & 0.1 & 0.23 & 0.38 & 0.5 & 0.61 \\
\hline Markazi & 0.48 & 0.48 & 0.5 & 0.5 & 0.5 & 0.5 \\
\hline Hormozgan & 0.49 & 0.13 & 0.15 & 0.49 & 0.5 & 0.5 \\
\hline Hamedan & 0.24 & 0.24 & 0.24 & 0.25 & 0.23 & 0.53 \\
\hline
\end{tabular}




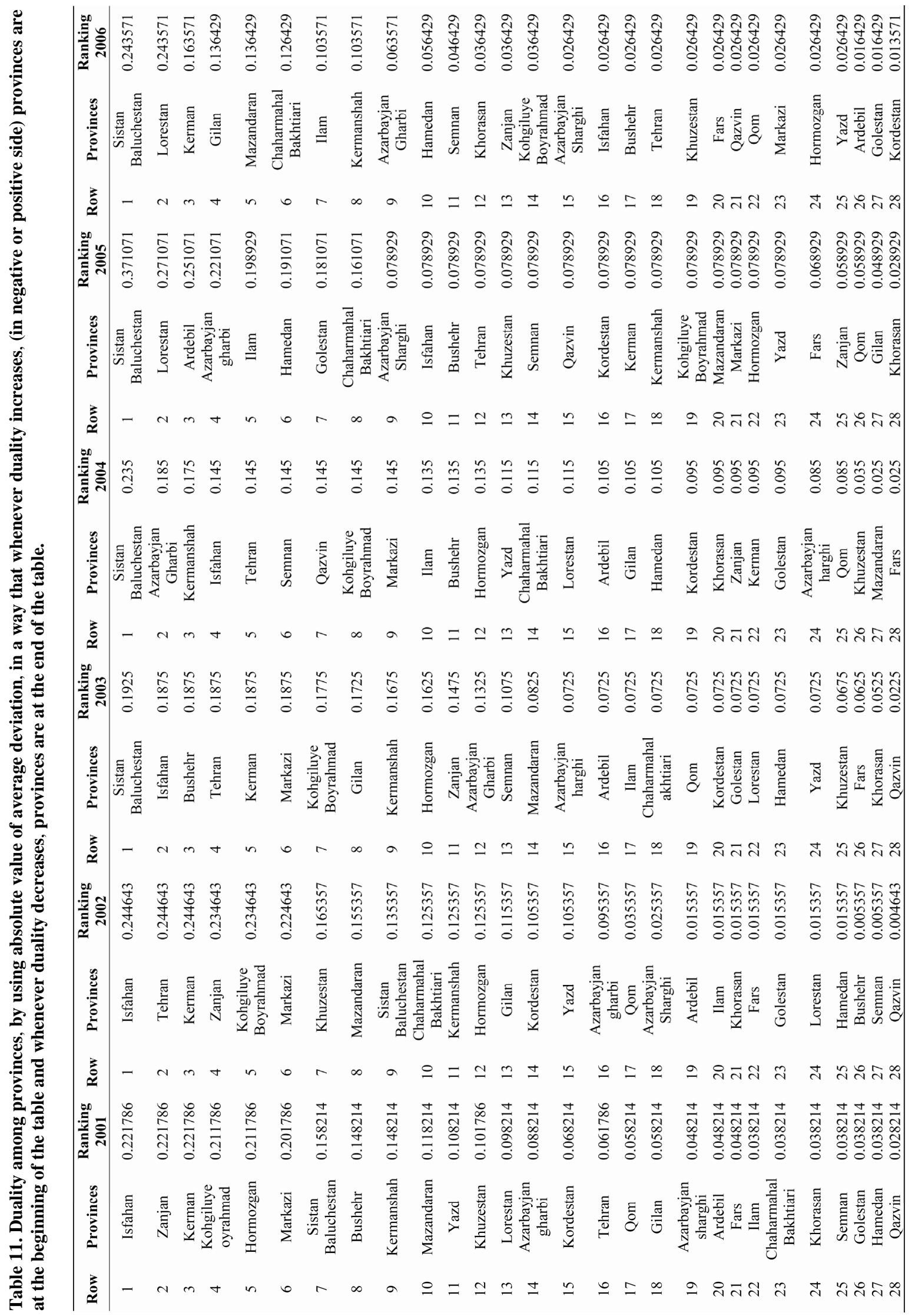


period (2001-2006), these differences decreased and provinces get closer to the average (Table 10). In other words, convergence increased among provinces. However, comparing provinces and their rankings revealed considerable differences and inequalities among provinces. Therefore, continuous further attempt and investigation to decrease these inequalities and move forward to sustainable development in all provinces is highly required. Ilam and Gilan during 2005 and 2006 and Isfahan, during 2001 to 2004, were the highest developed provinces. In contrast, Sistan Baluchistan during 2001, 2003, 2004 and 2005; Khuzestan during 2002 and Lorestan during 2006 were the lowest provinces (Table 10).

\section{Duality}

Duality is a valuable criterion for evaluating un-uniform movement of resources in the field of development [10]. In fact, duality can discover the gap among provinces, based on selected indices. In this work, duality set of different conditions, some of which are desirable and some are non-desirable were considered. If we consider desirable conditions as an average of sustainable development indices, in order to show the duality among provinces, absolute value of average deviation, is a reliable index.

Whenever the duality increases, (negative or positive side) provinces are at the beginning of the table, and whenever duality decreases, provinces are at the end of the table (Table 11). During the years of investigation (2001-2006), Isfahan and Sistan Baluchestan had the most duality (Table 11). Because of more movement of resources into Isfahan, its distance from the average has been increased in positive side. In contrast, in Sistan Baluchestan consideration movement of resources has occurred. As result, its distance from average has been increased in negative side. (Table 11). Figure 3 presents the differences among 6 provinces which have the highest and lowest ranking among 28 provinces of country

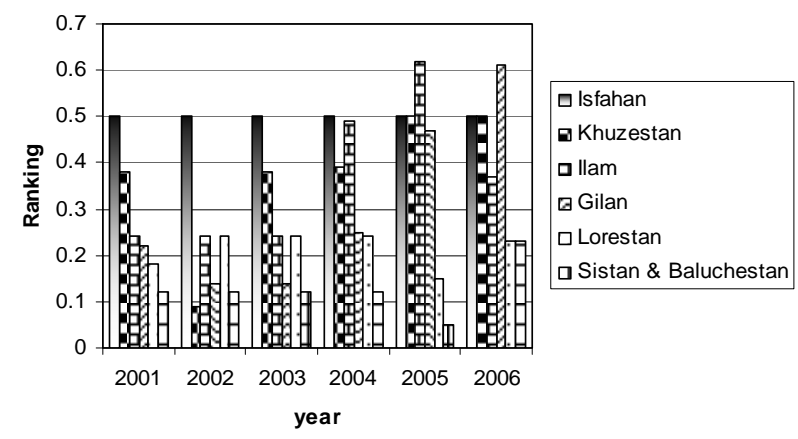

Figure 3. The differences among 6 provinces which have the highest and lowest ranking among 28 provinces of country during 6 years of under investigation. during the period of under investigation. As it can be inferred from Table 11, during the period of the study, the distance among the provinces has been decreased in relation to mean (overall average ) become more homogeneous. However, the absolute distance between provinces has been increased.

\section{Conclusions}

The results showed that during 6 years of investigation, some of the provinces were always at the end of the ranking (Table 11). We suggest that programmers and politicians should pay more attention to these provinces.

An interesting phenomenon was observed that changes in ranking of most of the provinces were not constant, and this is an indicator of lack of coordination and much variation in the programs of government (Table 10). It is suggested that programs should be compiled with accurate study and according to the facility of each province.

This study showed that in developing countries such as Iran, one development program cannot be effective for all areas of a country with socio-economic duality. In addition, this work showed that fuzzy system can efficiently be used in clustering of different un-uniform parts of country.

We suggest that in future works, in addition to fuzzy models, novel data mining methods such as decision tree and neural network algorithms can be considered to apply in development programming. In fact, due to complicated and un-uniform nature of sustainable development in developing countries, data mining has the potential of discovering previously unknown and potentially interesting patterns in large datasets based on socioeconomic indices [11]. In particular, feature selection (feature weighting) can dissect different socio- economic indices from each other clarifying the importance of indices by adding value to them [12]. As a result, more precise reliable developing program is probable.

\section{Acknowledgements}

We would like to thank Azad University for supporting this research. Also, we thank Dr. Esmaeil Ebrahimie (Shiraz University) for revising the manuscript and his valuable comments.

\section{References}

[1] T. Strange and A. Bayley, "Sustainable Development," Organization for Economic Co-operation and Development (OECD) Press, Paris, 2008.

[2] M. Bedrich and S. Billharz, "Sustainability Indicators," John Wiley \& Sons, Hoboken, 1997. 
[3] L.-X. Wang, "A Course in Fuzzy Systems and Control," Prentice Hall, Upper Saddle River, 1996.

[4] H. J. Zimmermann, "Fuzzy Set Theory,” Kluwer Academic Publishers, Norwell, 1996.

[5] Central Bank of Iran, “The Index of the Good's Price and Consumption Services in Urban Areas of Iran, 20012006,” 2011. http://tsd.cbi.ir/

[6] Statistical Centre of Iran, 2011. http://amar.sci.org.ir/

[7] The Management and Planning Organization of Iran (MPO), 2011. http://www.spac.ir/

[8] F. Noorbakhsh, "Human Development and Regional Disparities in Iran: A Policy Model,” Journal of International Development, Vol. 14, No. 7, October 2002, pp. 927-949. doi:10.1002/jid.914

[9] M. Ebrahimi, M. Noonejad and M. Ebrahimi, “ A Study of Iranian’s Free Zones Throughput; Success or Failure in
Achieving Proposed Goal?” Journal of Applied Sciences, Vol. 8, No. 8, 2008, pp. 1576-1580.

doi:10.3923/jas.2008.1576.1580

[10] M. Andreassen and F. Gertsen, “A Case Study of an Organisation Development of Duality," International Journal of Technology Management, Vol. 42, No. 1-2, 2008, pp. 51-68. doi:10.1504/IJTM.2008.018060

[11] M. Ebrahimi and E. Ebrahimie, "Sequence-Based Prediction of Enzyme Thermostability through Bioinformatics Algorithms," Current Bioinformatics, Vol. 5, No. 3, 2010, pp. 195-203. doi:10.2174/157489310792006693

[12] E. Bijanzadeh, Y. Emam and E. Ebrahimie, "Determining the Most Important Features Contributing to Wheat Grain Yield Using Supervised Feature Selection Model,” Australian Journal of Crop Science, Vol. 4, No. 6, 2010, pp. 402-407. 